\title{
On the Selection of Physical Layer Optimized Network Topologies for the Zigbee Network
}

\author{
Manpreet \\ Guru Nanak Dev University Regional Campus, Jalandhar, INDIA \\ E-mail: manpreetpawar17@gmail.com \\ Jyoteesh Malhotra \\ Guru Nanak Dev University Regional Campus, Jalandhar, INDIA \\ E-mail: jyoteesh@gmail.com
}

\begin{abstract}
Zigbee standard has been designed for low data rate, low cost and limited power applications for short-range wireless communication. The successful implementation of Zigbee based network depends on the suitable selection of physical (PHY) layer and medium access control (MAC) layer parameters. In this work the PHY layer parameters have been optimized for star, tree and mesh topologies. The performance investigations have been carried out for different frequency band and data rate and different bandwidth (BW) in each of standard topologies. Through extensive simulations, QoS parameters like throughput, network load and delay have been evaluated to achieve optimal performance of physical layer.
\end{abstract}

Index Terms-Zigbee, frequency band, data rate, bandwidth, star topology, mesh topology, tree.

\section{INTRODUCTION}

Zigbee technology is a wireless personal area network based on standard IEEE 802.15.4 targeted for wireless sensor applications due to its low data rate and low power consumption. It is widely used various applications from home appliances to military [1]. Zigbee is the enhancement of IEEE 802.15.4 as it introduced network and application layer on the top of IEEE 802.15.4 layered architecture which support two layers: PHY and MAC layer. [2]. Zigbee network specifies two types of devices: Full functional devices (FFD) and Reduced functional devices (RFD). FFDs (Coordinator and router) mainly do routing, sensing and coordination but RFDs (end devices) only perform sensing and transmit messages to FFD. There are basic three topologies: Star, tree and mesh. In star topology one coordinator connects to end devices. Tree topology maintains parent-child relationship in which role of root played by coordinator and can be act as the parent of routers, coordinators and routers can be parent in network and end devices can only be children. Mesh, most adaptable and trustworthy topology because there are number of potential paths exists for transmission of message [3].

Many authors made efforts to analyze the behavior of Zigbee topologies to make QoS provisioned topologies.
In [4] different packet reception value has been used resulted that mesh and tree topologies gave optimum performance on $-85 \mathrm{dBm}$ and mesh topology is most reliable topology in case of failure of any device as mesh topology show self healing mechanism. [5] Shown that MSK should be favored if there is need to maximize the SNR and QAM_64 is inappropriate for that case. BPSK suitable if there is need to minimize the packets marked noise at the radio receiver of PAN Coordinator and GTS End Device but in case of non GTS end devices MSK perform better than BPSK. [6] Performed simulation of Zigbee network on 64kbps, $128 \mathrm{kbps}$ and $250 \mathrm{kbps}$ and by varying power. The results showed varied relationship between the signal Bit Error Rate (BER) and Signal to Noise Ratio (SNR) when data rate and power varied. In [7] simulation of Zigbee and wi-fi network has been done by using the frequency band offset. The simulation results shows that a small offset can provide sufficient improvement in the performance of Zigbee network.

It has been found the work reported earlier to ptimize PHY layer limited to modulation key; transmit power, packet reception value. However frequency bands and data rate selection for reliable transmission needs to be considered. The work related to data rate, frequency bands for BER, SNR have been evaluated but other QoS parameters had not been taken. In this paper by work has been done by taking different frequency bands and data rate to evaluate QoS parameter like throughput, load, and delay.

The rest of the paper is organized as follows. Section II describes background of physical layer of Zigbee layered architecture. Section III describes the simulation methodology and environment used for extensive simulation. Section IV depicts the results and discussions of work to optimize the physical layer before the paper is concluded in section $\mathrm{V}$.

\section{BACKGOUND}

The physical layer is provided by the IEEE 802.15.4 standard. It is very close to hardware. The characteristics of the PHY are opening and closing of the radio transceiver, energy detection (ED), channel selection, clear channel assessment (CCA), link quality indication 
(LQI) and dispatch as well as obtain packets across the physical medium. Each device usually transmits with $1 \mathrm{~mW}$ but if there is lower need of power then power can be less than $1 \mathrm{~mW}$ [8].

This standard deals with the physical transmission of radio waves in different unlicensed frequency bands around the world to provide communication between devices within a WPAN. These frequency bands support different channels: 16 channels available in $2.4 \mathrm{GHz}$ band with $250 \mathrm{kbps}$ data rate, 10 channels available in 902-928 $\mathrm{MHz}$ band with $40 \mathrm{kbps}$ data rate and only 1 channel available in $868-870 \mathrm{MHz}$ band provide with $20 \mathrm{kbps}$ data rate. The $868 / 915 \mathrm{MHz}$ PHY uses a DSSS approach, in this every send out bit represented by 15-chip maximum length sequence. To encode the binary code, 1 or -1 is multiplied and resulting sequence modulated onto carrier using binary phase shift key (BPSK). 2.4 GHZ PHY uses 16-ary quasi-orthogonal modulation (based on DSSS). In this chip sequence is modulated on carrier using offset-quadrature phase shift keying (OQPSK) [9].

\section{Simulation Methodology AND ENVIRONMENT}

For the simulation tool, OPNET [10] and NS-2 [11] are the most useful tools to simulate the different protocols. Because NS-2 was at first developed for IP networks and then widened for IEEE 802.11 wireless networks so OPNET have more capability to simulate the ZigBee protocol more perfectly without excessive overheads [12] Following figure 1 to figure 6 are the simulation environments used for investigation.

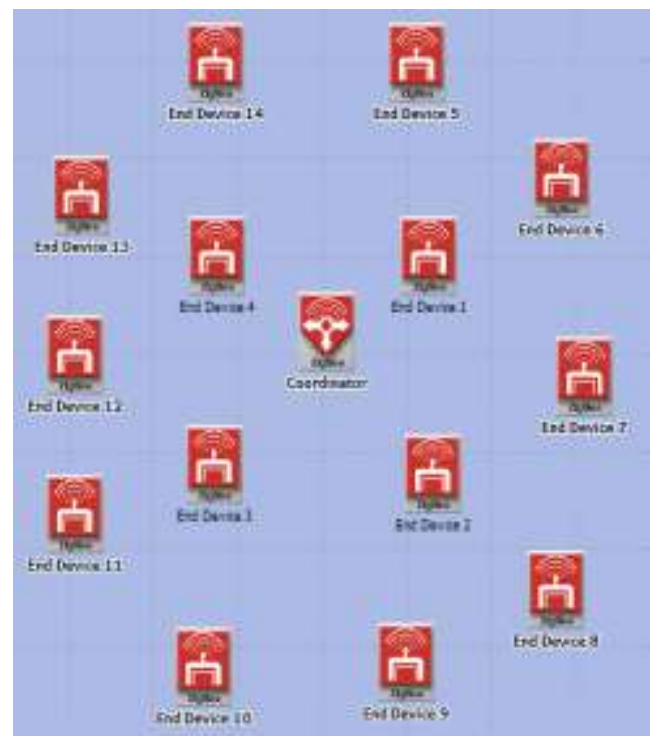

Fig.1. Star Topology (15 nodes)

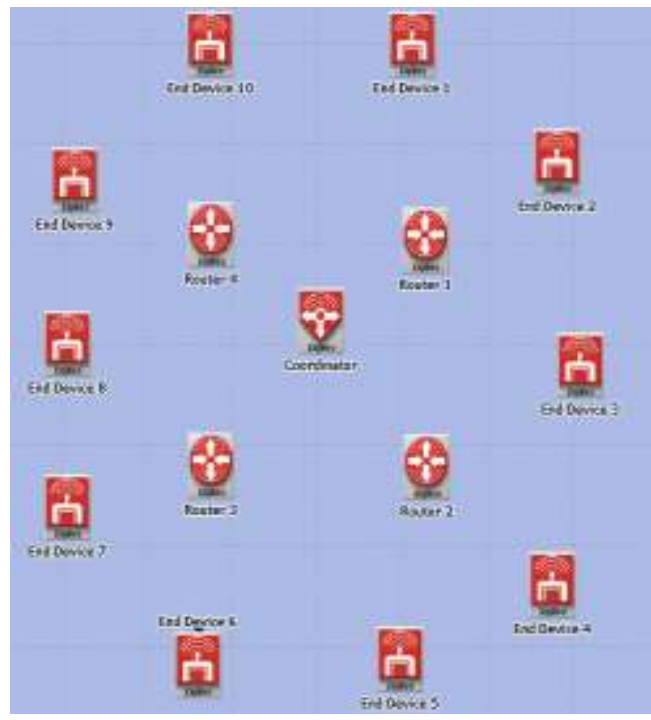

Fig.2. Tree Topology (15 nodes)

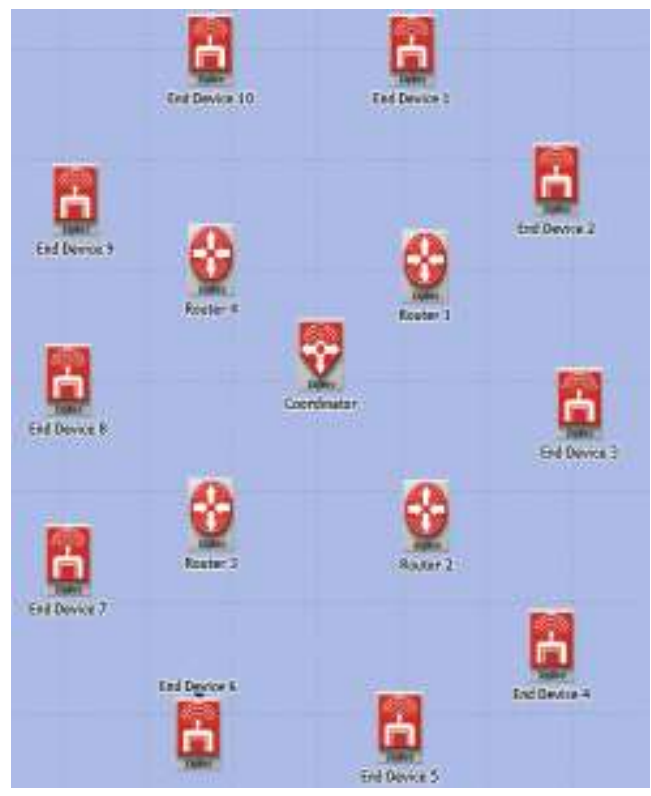

Fig.3. Mesh Topology (15 nodes)

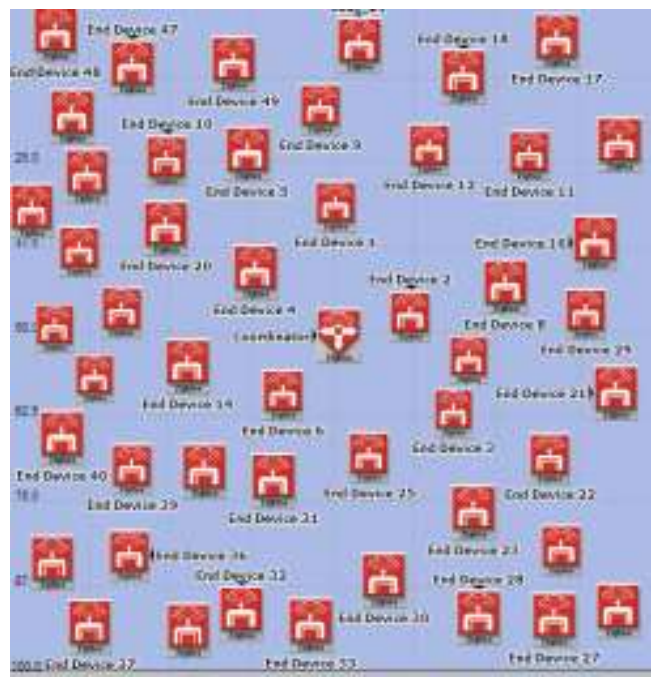

Fig.4. Star Topology (50 nodes) 


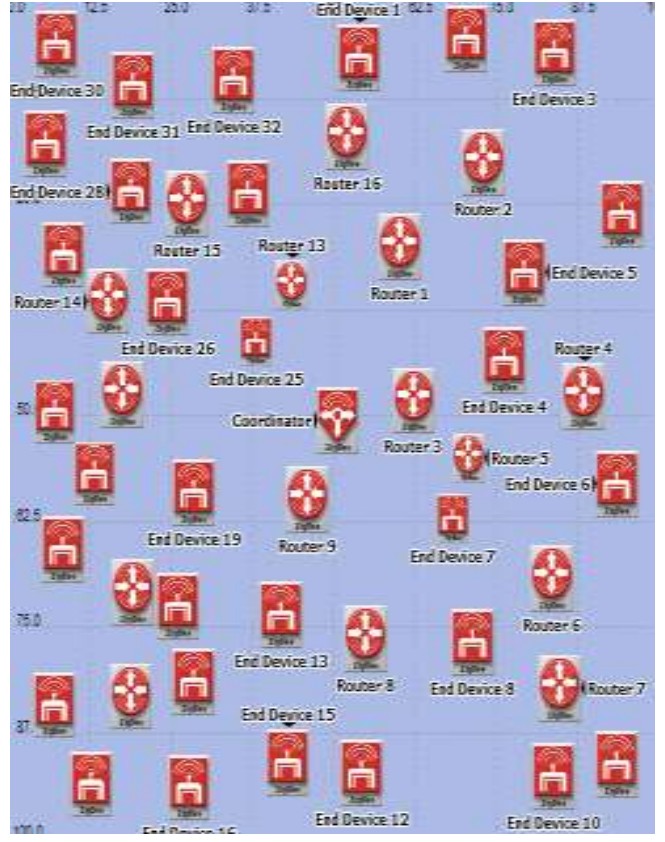

Fig.5. Tree Topology (50 nodes)

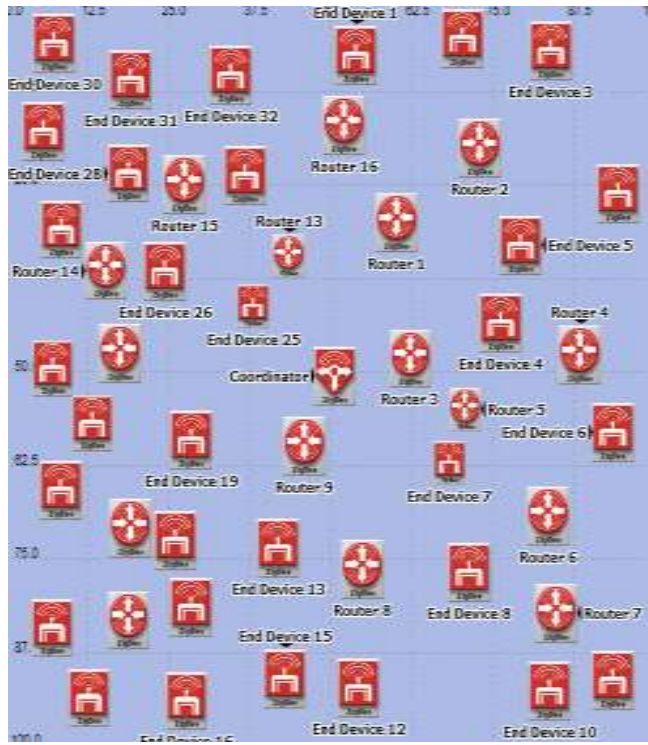

Fig.6. Mesh Topology (50 nodes)

In each of set up $100 * 100$ meters has been taken. For 15 nodes network, in star topology coordinator is surrounded by 14 end devices and in tree and mesh topologies 1 coordinator, 4 router and 10 end devices have been set up. For 50 nodes network, in star topology coordinator is surrounded by 49 end devices and in tree and mesh topologies 1 coordinator, 16 router and 33 end devices have been set up In Table I different values of parameter has been described which are used during simulation analyses.

The main consideration to check the effect of frequency bands and suitable data rate for the topologies, the Frequency band and data rate parameters have been carried in the PHY layer. Firstly results are taken on
2.4GHz and then frequency band has been set to $915 \mathrm{MHz}$ for star, tree and mesh topologies which is followed by evaluation of different data rates such as $50 \mathrm{kbps}, 100 \mathrm{kbps}$ and $150 \mathrm{kbps}$ to know the effect of less and more data rate on topologies. After that $10 \mathrm{kHz}$ and $100 \mathrm{kHz} \mathrm{BW}$ size has been taken to evaluate the effect of BW on small network size and large network size.

Table 1. Parameters And Values

\begin{tabular}{|c|c|}
\hline Parameters & Value \\
\hline Area & $100 * 100$ meters \\
\hline \multicolumn{2}{|c|}{ MAC Layer Parameters } \\
\hline$\overline{\mathrm{ACK}}$ & Enabled \\
\hline ACK wait Duration & 0.05 seconds \\
\hline No. of retransmission & 5 \\
\hline CSMA & Enabled \\
\hline Minimum Back-off Exponent & 3 \\
\hline Maximum number of Back-offs & 4 \\
\hline Channel Sense Duration & 0.03 seconds \\
\hline \multicolumn{2}{|c|}{ PHY Layer Parameters } \\
\hline Data rate & $\begin{array}{l}50 \mathrm{kbps}, 100 \mathrm{kbps} \text {, } \\
150 \mathrm{kbps}\end{array}$ \\
\hline Frequency band & $2.4 \mathrm{GHz}, 915 \mathrm{MHz}$ \\
\hline $\begin{array}{lll}\begin{array}{l}\text { Packet } \\
\text { threshold }\end{array} & \text { reception } & \text { power } \\
\end{array}$ & $-85 \mathrm{dBm}$ \\
\hline Transmit Power & $0.05 \mathrm{~W}$ \\
\hline Modulation key & BPSK \\
\hline BW & $10 \mathrm{kHz}, 100 \mathrm{kHz}$ \\
\hline \multicolumn{2}{|c|}{ Application Layer Parameters } \\
\hline Destination & Random \\
\hline Packet Inter-arrival time & Constant(1) \\
\hline Packet Size & Constant(1024) \\
\hline Start Time & Uniform $(20,22)$ \\
\hline Stop Time & infinity \\
\hline
\end{tabular}

\section{RESULT AND DISCUSSION}

Based on simulation scenario developed in the previous section, extensive simulation has been done and the results are obtained. In this section the relative performance of merits and demerits of star, tree and mesh topologies have been discussed in terms of throughput, load, data traffic sent, data traffic received and delay. Results obtained have been categorized based on frequency band, data rate and bandwidth.

It is observed from the Fig. 7 that for $2450 \mathrm{MHz}$, tree topology gives highest throughput (45860 bits/sec) and throughput of star topology is intermediate (31320 bits/sec) and star topology give least throughput (29290 bits/sec). Figure 3 that for $914 \mathrm{MHz}$, throughput of tree topology falls (29500 bits/sec) and throughput of mesh topology show minor decrease $(28500 \mathrm{bits} / \mathrm{sec})$ in 915 $\mathrm{MHz}$ frequency band and star topology also show decrease in throughput (30200 bits/sec). 


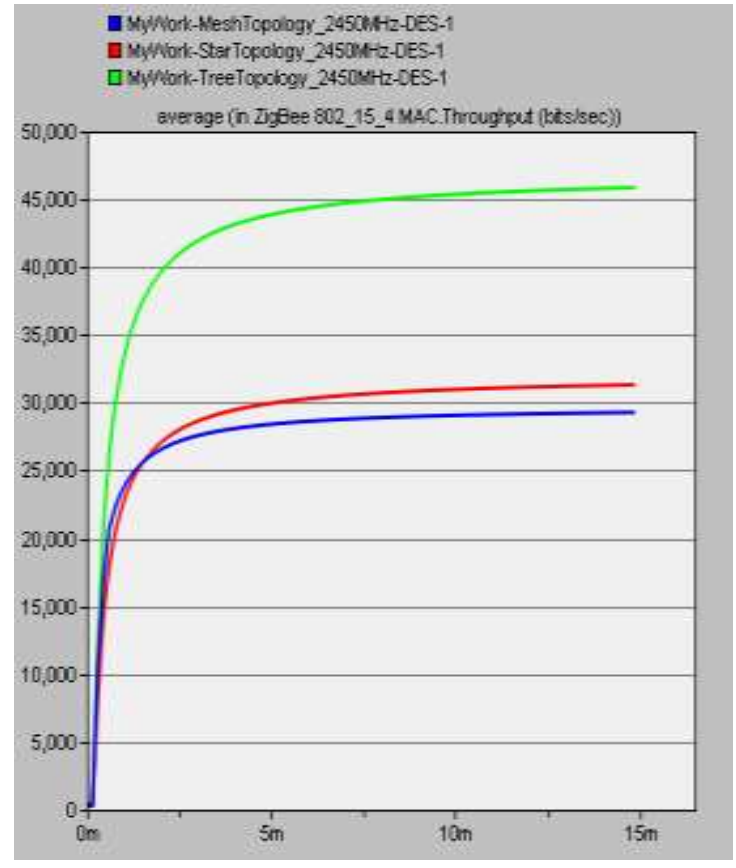

Fig.7. Throughput (2.4GHz)

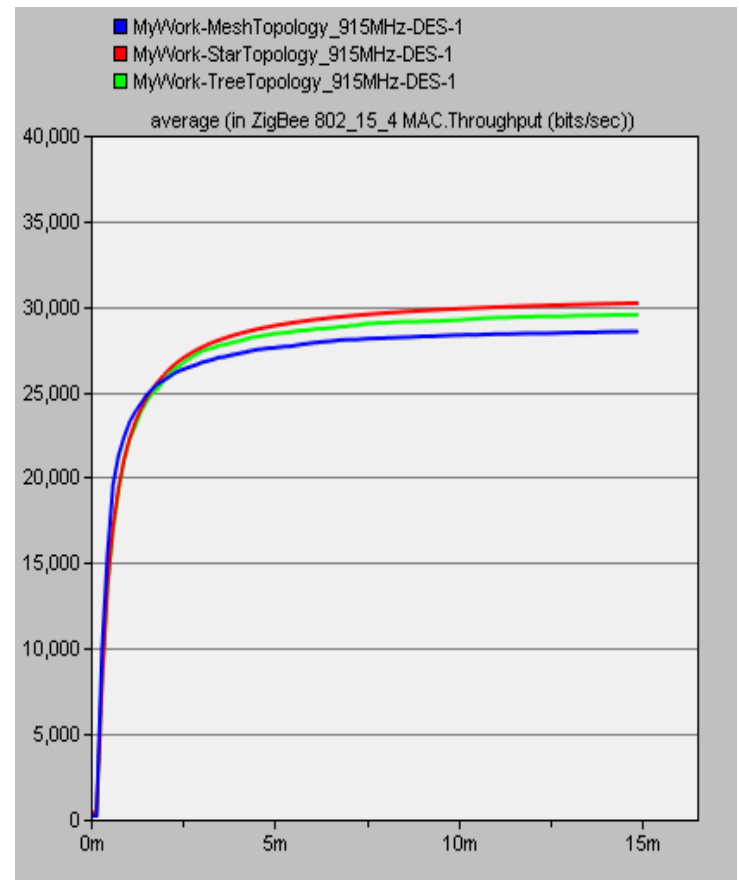

Fig.8. Throughput (915MHz)

For $2.4 \mathrm{GHz}$ band, load is less in mesh topology (29110 bits/sec). Load of star topology is more than load in mesh topology (32440 bits/sec) but tree topology has highest load (45860 bits/sec). It is observes in figure for $915 \mathrm{MHz}$, load of star topology decreases as from load for $2.4 \mathrm{GHz}$ and load in mesh topology increases. Load for tree topology (31850 bits/sec) is less as compared to $2.4 \mathrm{GHz}$.

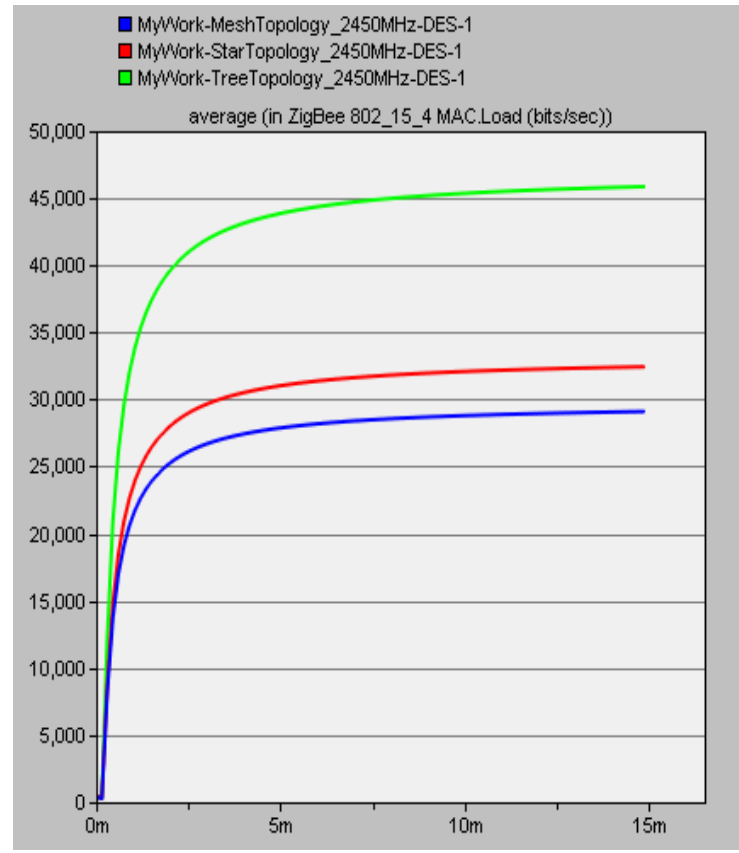

Fig.9. Load (2.4GHz)

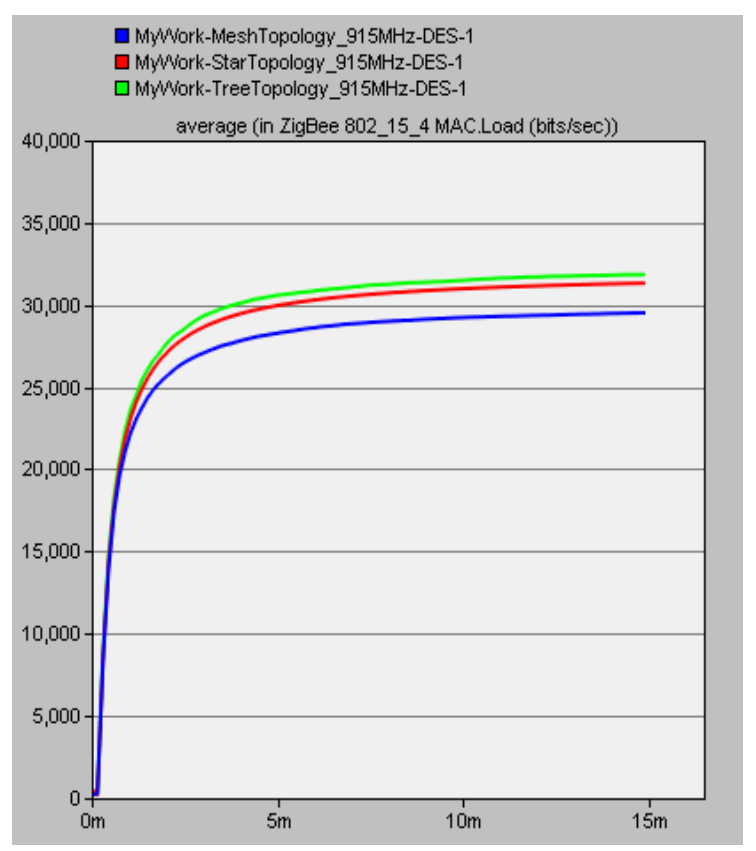

Fig.10. Load (915MHz)

For $2.4 \mathrm{GHz}$ delay slightly different in all the cases with minor variations. Delay in tree topology is 0.0103 sec and delay in star topology is $0.0095 \mathrm{sec}$ and delay in mesh topology is $0.009 \mathrm{sec}$. For $915 \mathrm{MHz}$, tree topology is showing maximum delay as $0.15 \mathrm{sec}$ and in mesh and star delay is $0.083 \mathrm{sec}$ and $0.104 \mathrm{sec}$ respectively. 


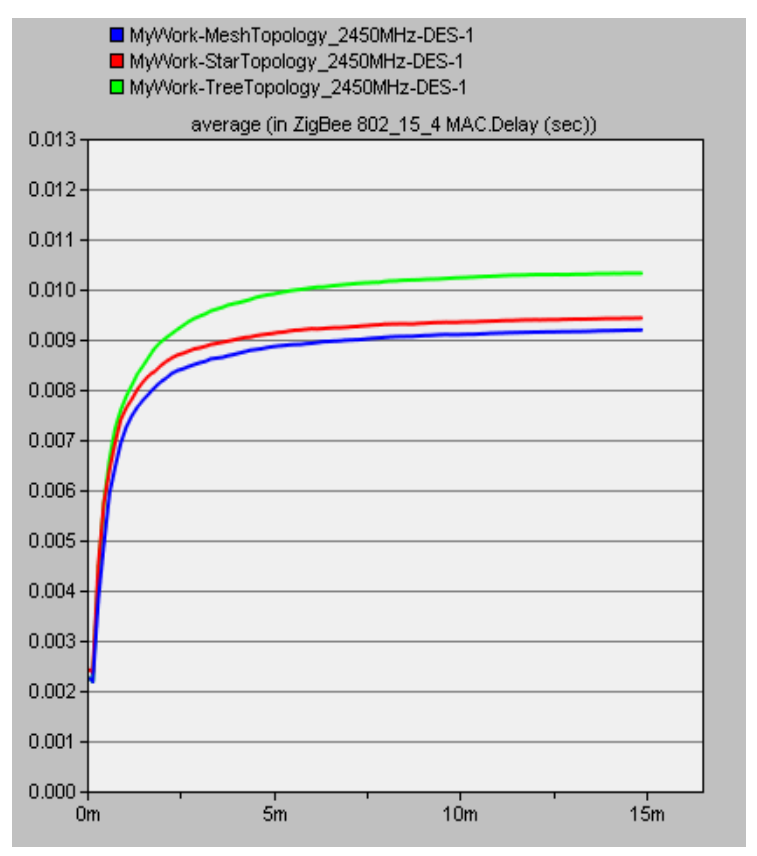

Fig.11. Delay $(2.4 \mathrm{GHz})$

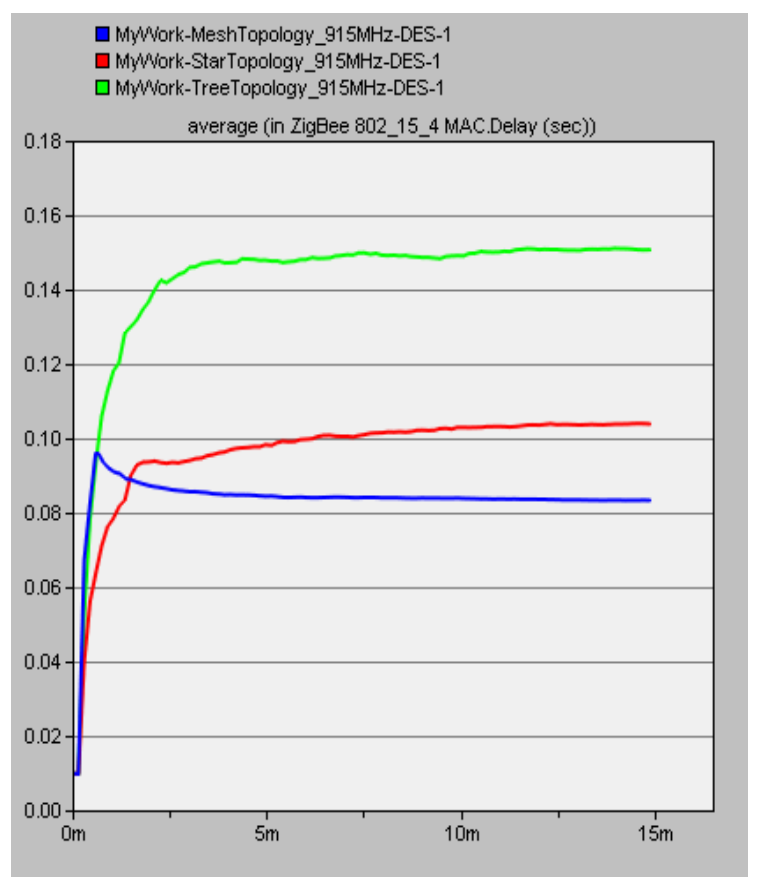

Fig.12. Delay (915MHz)

Results show that Star topology show minor decrease in performance for throughput, load, and data traffic sent and data traffic received in $915 \mathrm{MHz}$ as compared to 2.4 $\mathrm{GHz}$ frequency band. Tree topology outperforms in $2.4 \mathrm{GHz}$ frequency band and mesh topology perform better as compared to performance for $915 \mathrm{MHz}$. For 915 $\mathrm{MHz}$, all topologies show enormous increase delay as compared to delay at $2.4 \mathrm{GHz}$ as shown in Table II.
Table.2. Performance of Zigbee Topologies for $915 \mathrm{MHz}$ and $2.4 \mathrm{Ghz}$ Frequency Band

\begin{tabular}{|c|c|c|c|c|c|c|}
\hline $\begin{array}{c}\text { Resulted } \\
\text { Parameters } \\
\text { (Maximum }\end{array}$ & \multicolumn{2}{|c|}{ Frequency Band $=2.4$ GHz } & \multicolumn{3}{|c|}{$\begin{array}{c}\text { Frequency Band }= \\
\text { 915MHz }\end{array}$} \\
\cline { 2 - 7 } Average) & Star & Tree & Mesh & Star & Tree & Mesh \\
\hline $\begin{array}{c}\text { Throughput } \\
\text { (bits/sec) }\end{array}$ & 31320 & 45860 & 29290 & 30200 & 29500 & 28500 \\
\hline $\begin{array}{c}\text { Load } \\
\text { (bits/sec) }\end{array}$ & 32440 & 45860 & 29110 & 31320 & 31850 & 29500 \\
\hline $\begin{array}{c}\text { Delay } \\
\text { (seconds) }\end{array}$ & 0.0094 & 0.00103 & 0.00918 & 0.104 & 0.15 & 0.083 \\
\hline
\end{tabular}

It can be seen from the Fig. 13 that for $50 \mathrm{kbps}$, tree topology gives highest throughput (about $33800 \mathrm{bits} / \mathrm{sec}$ ) and throughput of mesh topology (32600 bits/sec) and star topology give least throughput (30500 bits/sec). By increasing data rate more than $100 \mathrm{kbps}$ throughput of mesh increased (38300 bits/sec) and throughput of tree topology also increased (49000 bits/sec) and fluctuation in throughput of both topologies mesh and tree decreased

But further increase in data rate decreases the throughput of tree and mesh topologies. Throughput of star topology remains same for $100 \mathrm{kbps}$ and $150 \mathrm{kbps}$.

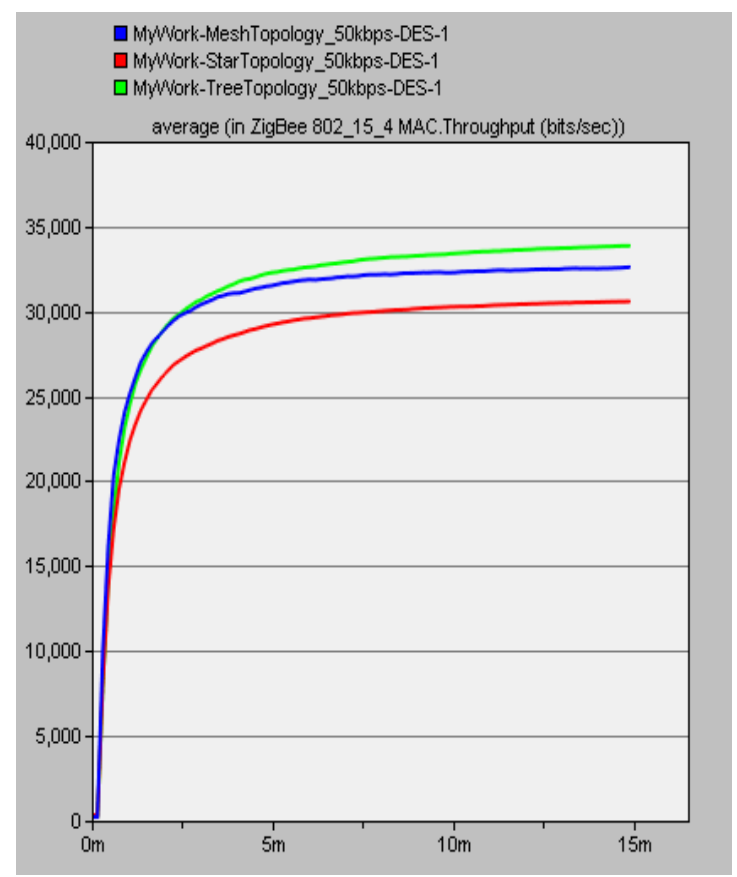

Fig.13. Throughput (50kbps) 


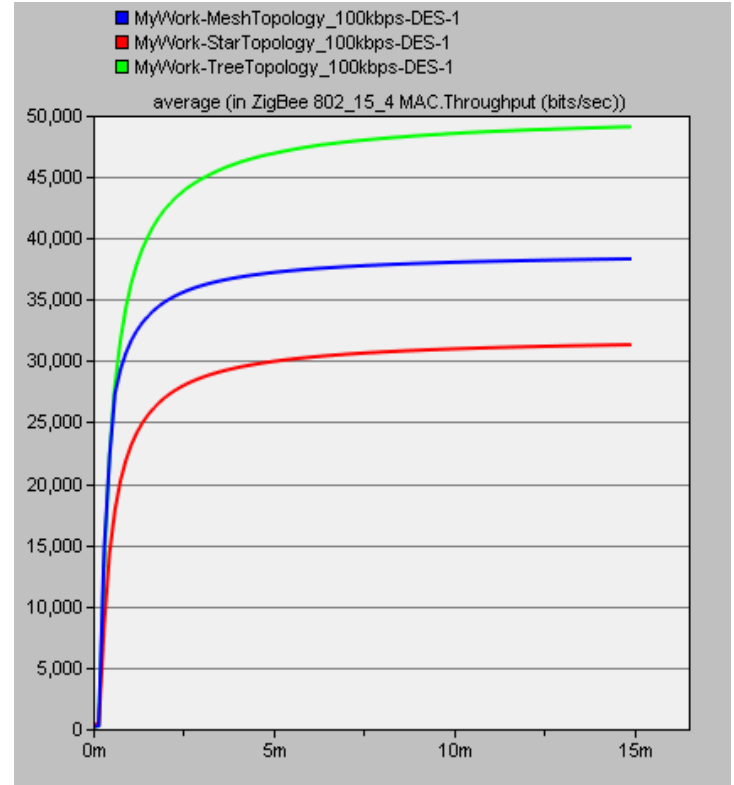

Fig.14. Throughput (100kbps)

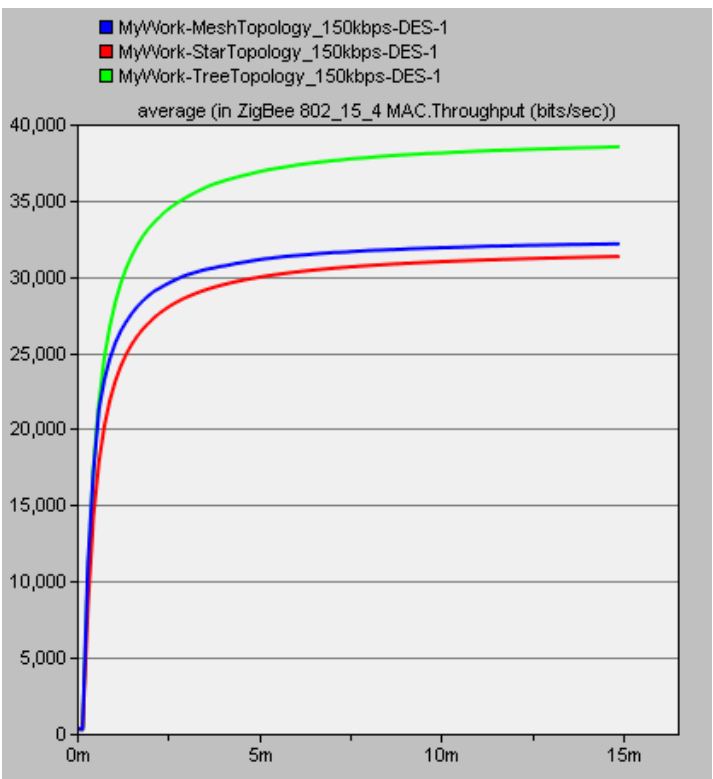

Fig.15. Throughput (150kbps)

It can be seen from the below figures that for $50 \mathrm{kbps}$, tree topology show highest load (about $36200 \mathrm{bits} / \mathrm{sec}$ ) but show minor fluctuation and load of mesh topology (33600 bits/sec) also fluctuate and star topology give least load (32400 bits/sec). By increasing data rate to $100 \mathrm{kbps}$ load of both topologies mesh and tree show increment and do not show fluctuation. But further increase in data rate decrease the load of tree and mesh topologies. Load of star topology show minor change by varying data rate from $50 \mathrm{kbps}$ to $100 \mathrm{kbps}$ but further increase in data rate do not affect the load in star topology.

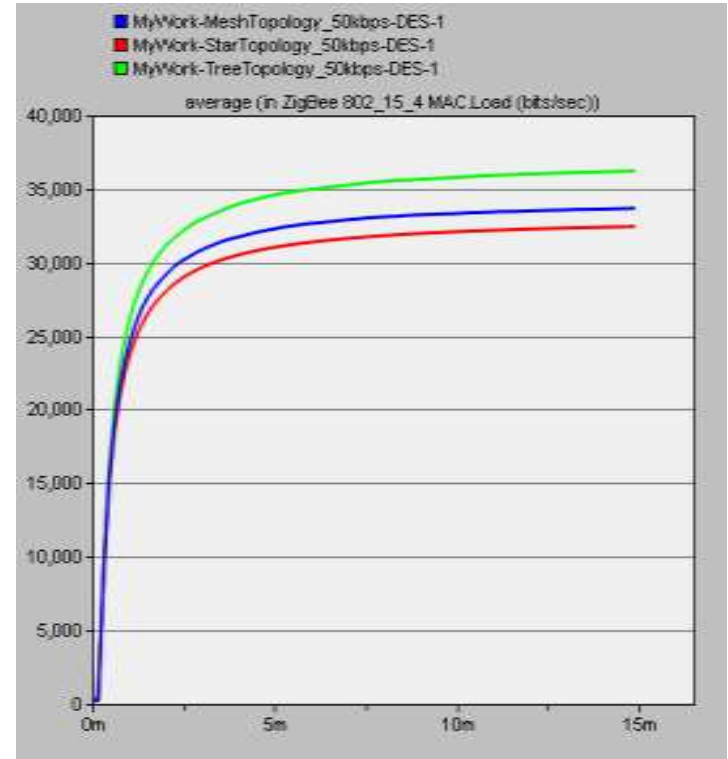

Fig.16. Load (50kbps)

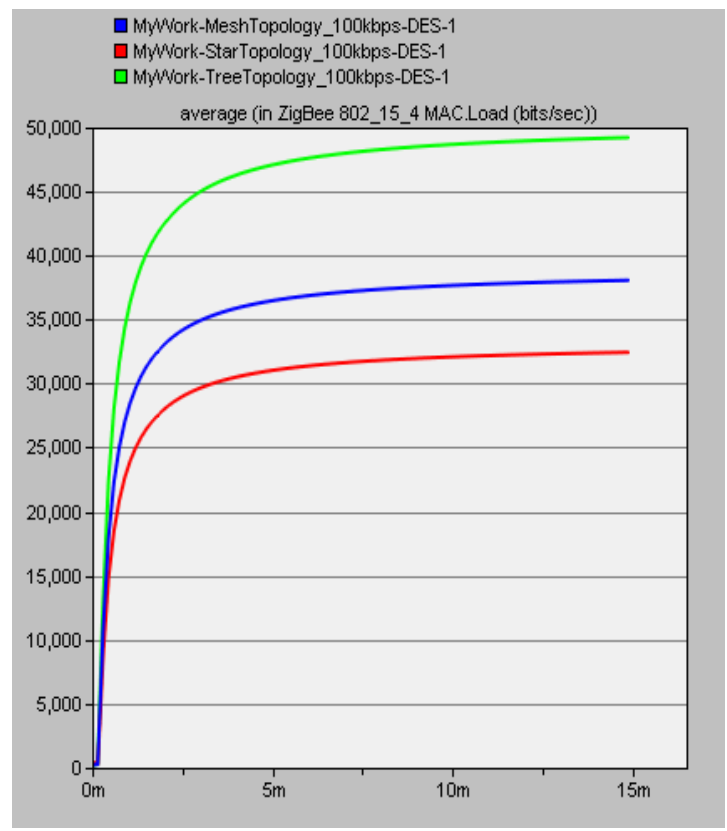

Fig.17. Load (100kbps) 


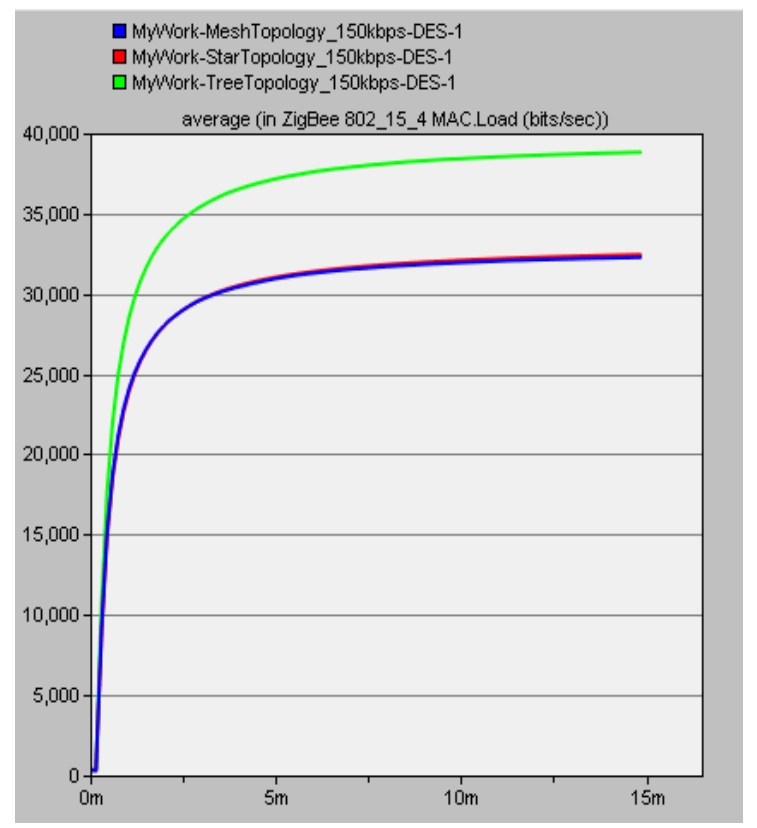

Fig.18. Load (150kbps)

Data traffic sent for 50kbps is highest in tree topology (39700 bits/sec) but show minor fluctuation and data traffic sent of mesh topology (37900 bits/sec) is moderate and show fluctuation and star topology have least data traffic sent (35600 bits/sec). By increasing data rate to $100 \mathrm{kbps}$, data traffic sent of both topologies mesh and tree increase and do not show fluctuation. But further increase in data rate, decrease the data traffic sent of tree and mesh topologies. Data traffic sent of star topology remains show minor variation.

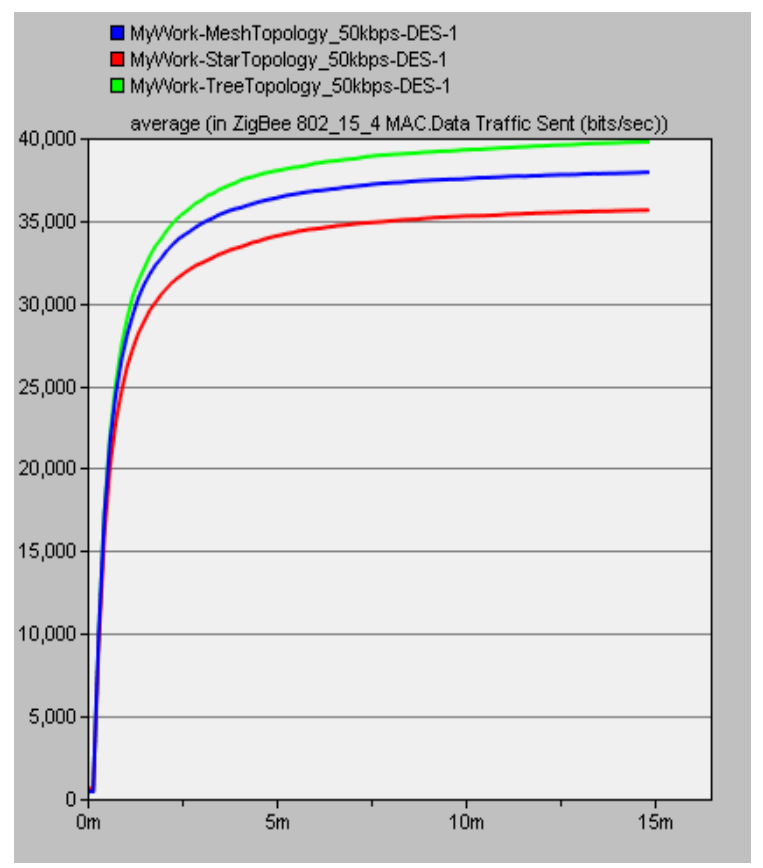

Fig.19. Data Traffic Sent (50kbps)

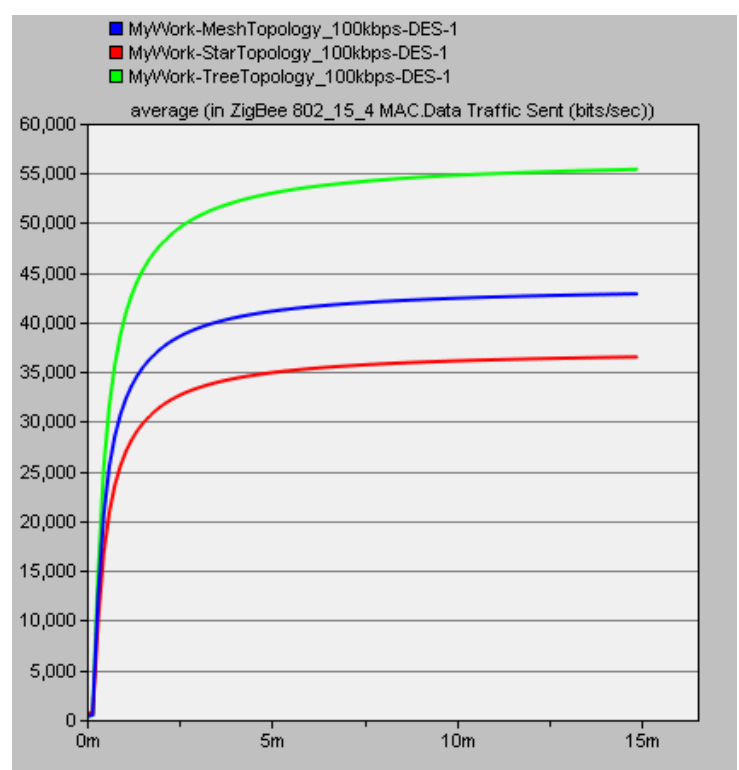

Fig.20. Data Traffic Sent (100kbps)

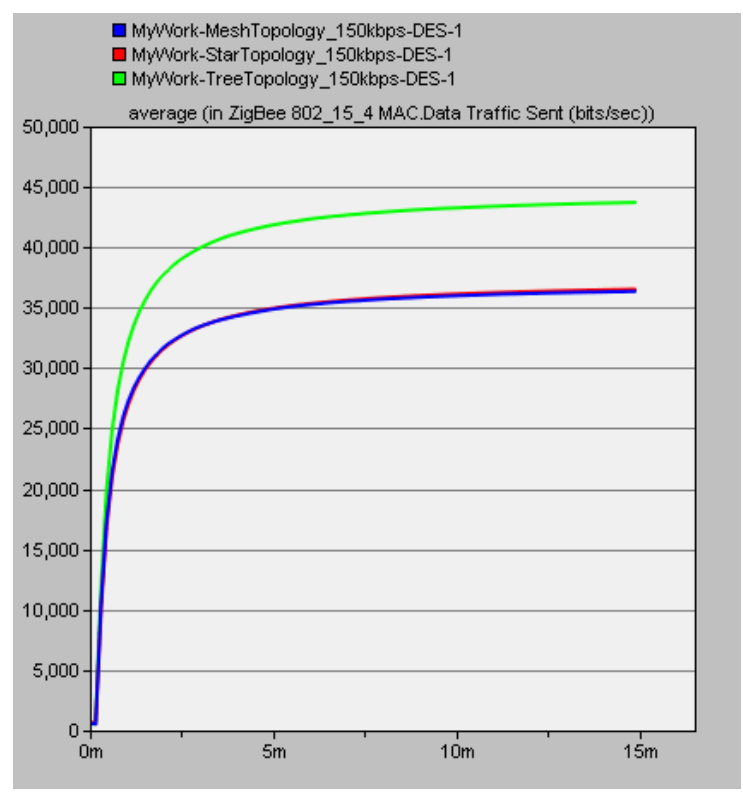

Fig.21. Data Traffic Sent (150kbps)

All the results show that star topology have minor effect by varying data rate from $50 \mathrm{kbps}$ to $100 \mathrm{kbps}$ and tree and mesh topologies perform better for data rate $100 \mathrm{kbps}$. Further increase in data rate fall the performance of tree and mesh topologies. All results have been summarized in Table III. 
Table 3. Performance of Zigbee topologies by varying data rate

\begin{tabular}{|c|c|c|c|c|c|c|c|c|c|}
\hline \multirow{2}{*}{$\begin{array}{c}\text { Resulted } \\
\text { Parameters }\end{array}$} & \multicolumn{3}{|c|}{ Data Rate = 50kbps } & \multicolumn{3}{c|}{ Data Rate =100kbps } & \multicolumn{3}{c|}{ Data Rate = 150kbps } \\
\cline { 2 - 10 } & Star & Tree & Mesh & Star & Tree & Mesh & Star & Tree & Mesh \\
\hline $\begin{array}{c}\text { Throughput } \\
\text { (bits/sec) }\end{array}$ & 30500 & 33800 & 32600 & 31300 & 49000 & 38300 & 31300 & 38500 & 32100 \\
\hline $\begin{array}{c}\text { Load } \\
\text { (bits/sec) }\end{array}$ & 32400 & 36200 & 33600 & 32400 & 49200 & 38000 & 32400 & 38000 & 32200 \\
\hline $\begin{array}{c}\text { Data Traffic } \\
\text { Sent } \\
\text { (bits/sec) }\end{array}$ & 35600 & 39700 & 37900 & 36500 & 55400 & 42800 & 36500 & 43700 & 36300 \\
\hline $\begin{array}{c}\text { Data Traffic } \\
\text { Received } \\
\text { (bits/sec) }\end{array}$ & 498800 & 513700 & 492700 & 510000 & 773000 & 600000 & 510000 & 584000 & 486000 \\
\hline
\end{tabular}

In this 2 network size have been taken one is of 15 nodes and one is of 50 nodes. QoS parameters such as throughput and delay have been evaluated by varying BW as $10 \mathrm{kHz}$ and $100 \mathrm{kHz}$.

It can be seen from the figure that for small network (15 nodes) there is no change in throughput in each topology by varying BW. Tree topology has highest throughput $(45850 \mathrm{bits} / \mathrm{sec})$ for each BW size. Star topology is showing intermediate throughput (31300 bits/sec) for each BW and mesh topology is showing lowest throughput (29200 bits/sec) for each topology. For large network (50 nodes), when BW increases from 10 $\mathrm{kHz}$ to $100 \mathrm{kHz}$ then throughput increases in tree (from $181500 \mathrm{bits} / \mathrm{sec}$ to $186500 \mathrm{bits} / \mathrm{sec}$ ) and mesh topologies (113600 bits/sec to $122200 \mathrm{bits} / \mathrm{sec})$. In star topology throughput (108500 bits/sec) remain same for $10 \mathrm{kHz}$ and $100 \mathrm{kHz}$.

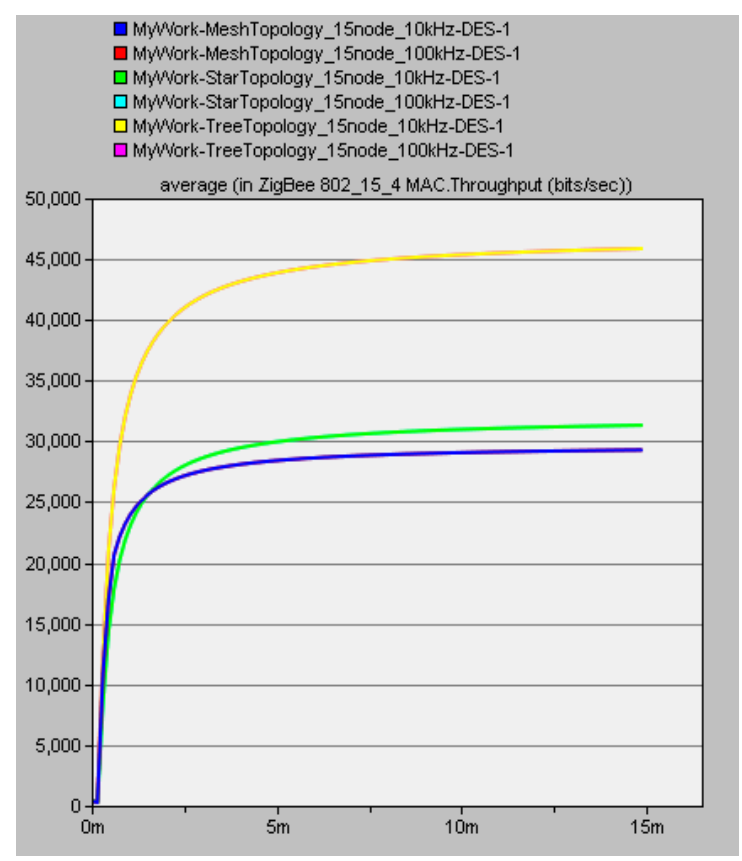

Fig.22. Throughput at $10 \mathrm{kHz}$ and $100 \mathrm{kHz}$ (15 nodes)

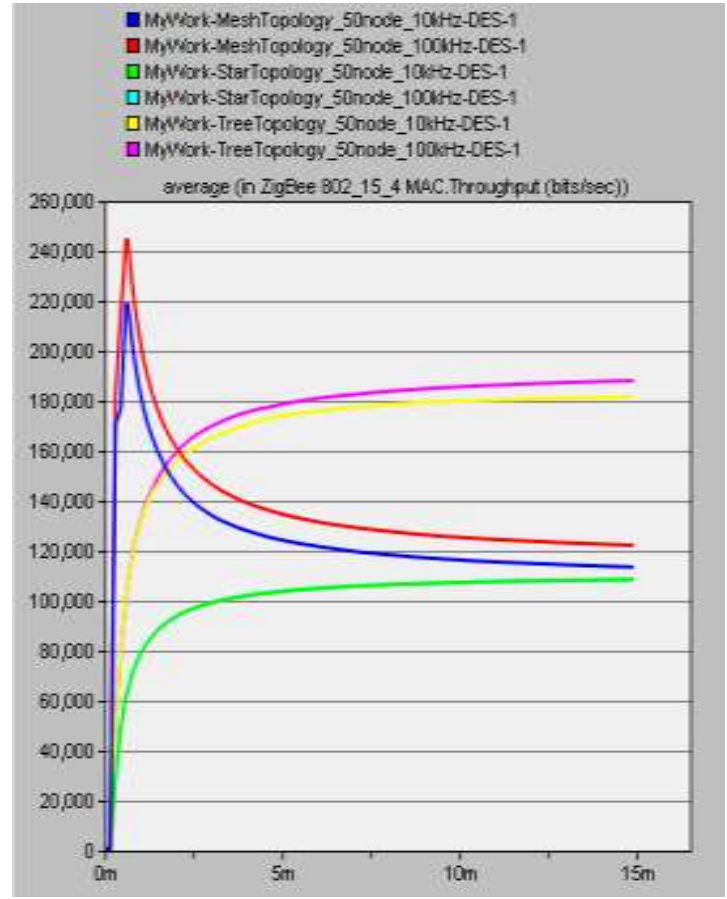

Fig.23. Throughput at $10 \mathrm{kHz}$ and $100 \mathrm{kHz}$ (50 nodes)

As shown in figure that for small network (15 nodes) there is no change in load in each topology by varying BW. Tree topology has highest load (45850 bits/sec) for each BW size. Star topology is showing intermediate load (32400 bits/sec) for each BW and tree topology is showing lowest load (45850 bits/sec) for each topology. But for 50 nodes network, each topology is showing different load. For large network (50 nodes), when BW increases from $10 \mathrm{kHz}$ to $100 \mathrm{kHz}$ then load increases in tree (from $193800 \mathrm{bits} / \mathrm{sec}$ to $201300 \mathrm{bits} / \mathrm{sec}$ ) and in mesh topologies (109100 bits/sec to $115200 \mathrm{bits} / \mathrm{sec}$ ). In star topology load (109600 bits/sec) remain same for 10 $\mathrm{kHz}$ and $100 \mathrm{kHz}$. 


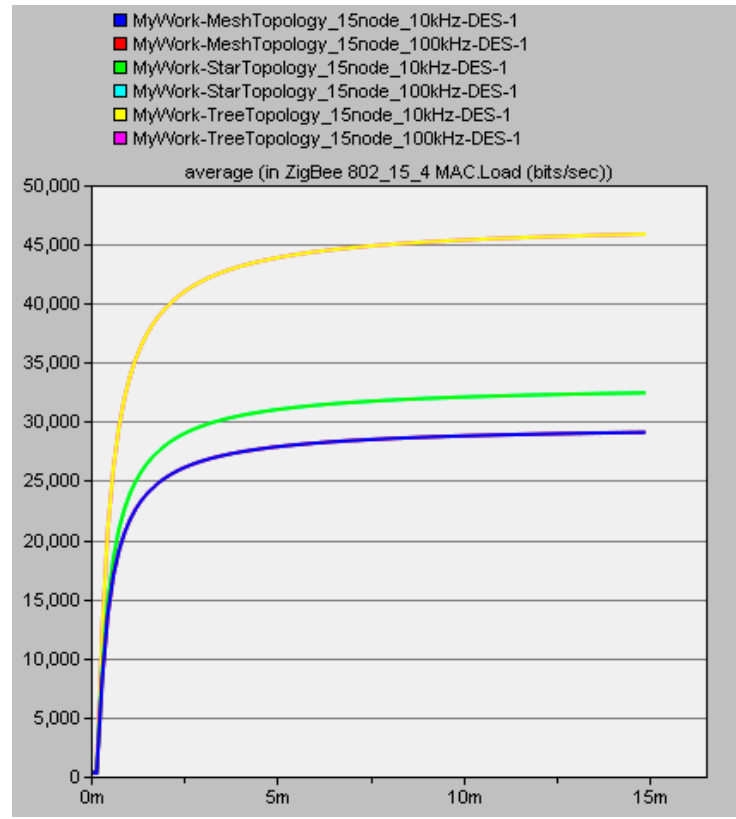

Fig.24. Load at $10 \mathrm{kHz}$ and $100 \mathrm{kHz}$ (15 nodes)

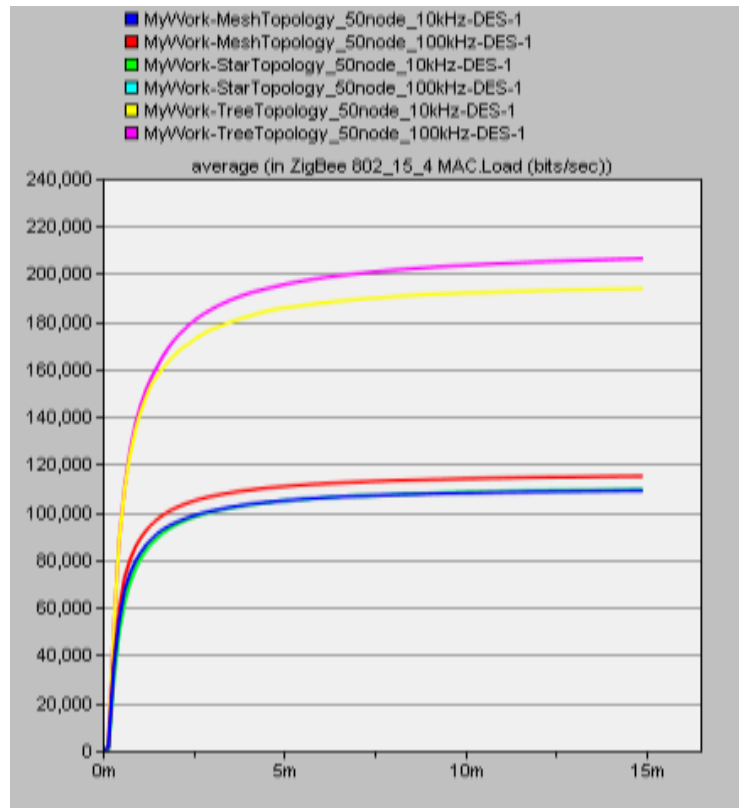

Fig.25. Load at $10 \mathrm{kHz}$ and $100 \mathrm{kHz}$ (50 nodes)

It can be seen from the figure that for small network (15 nodes) there is no change in data traffic sent in each topology by varying BW. Tree topology has highest data traffic sent $(51640 \mathrm{bits} / \mathrm{sec})$ for each $\mathrm{BW}$ size. Star topology is showing intermediate data traffic sent (36500 bits/sec) for each BW and mesh topology is showing lowest data traffic sent (32790 bits/sec) for each topology. For large network (50 nodes), when BW increases from $10 \mathrm{kHz}$ to $100 \mathrm{kHz}$ then data traffic sent increases in tree (from $218200 \mathrm{bits} / \mathrm{sec}$ to $221600 \mathrm{bits} / \mathrm{sec}$ ) and mesh topologies (123000 bits/sec to $129900 \mathrm{bits} / \mathrm{sec})$. In star topology data traffic sent (123400 bits/sec) remain same for $10 \mathrm{kHz}$ and $100 \mathrm{kHz}$.

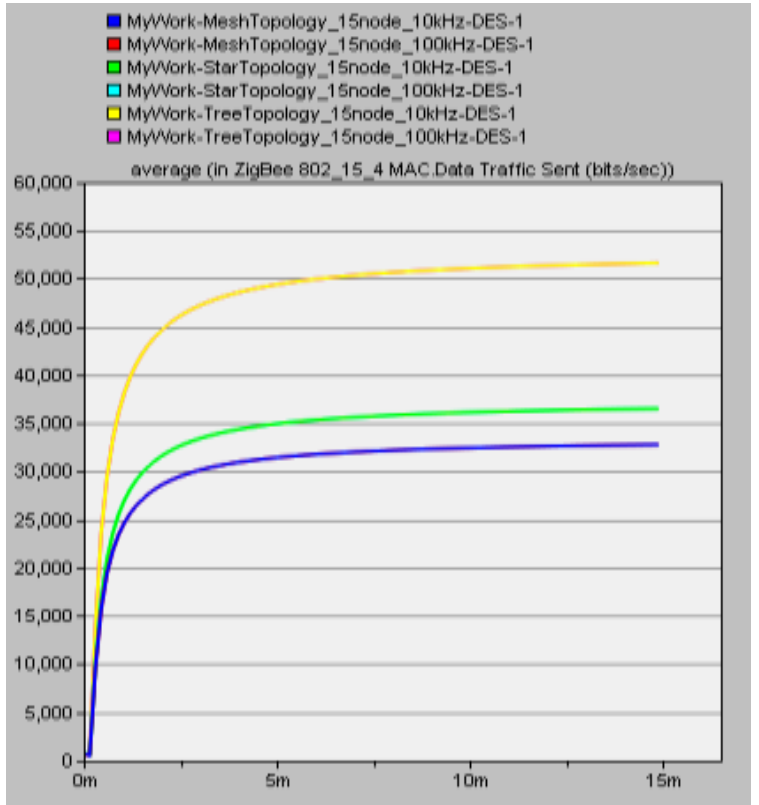

Fig.26. Data Traffic Sent at $10 \mathrm{kHz}$ and $100 \mathrm{kHz}$ (15 nodes)

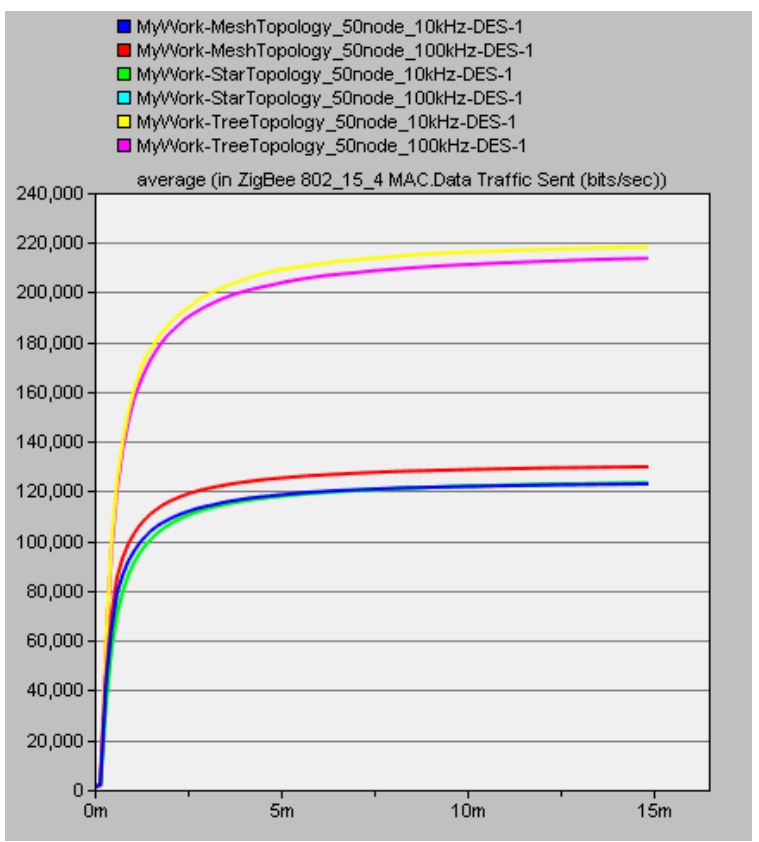

Fig.27. Data Traffic Sent at $10 \mathrm{kHz}$ and $100 \mathrm{kHz}$ (50 nodes)

All the results show that star topology has no effect by varying BW. Throughput, network load, data traffic sent and data traffic received increase in tree and mesh topologies by increasing network size and BW. All results have been summarized in Table III. 
Table 4. Performance of Zigbee topologies by varying Bandwidth

\begin{tabular}{|c|c|c|c|c|c|c|}
\hline \multicolumn{7}{|c|}{ Network Size $=15$ nodes } \\
\hline & \multicolumn{3}{|c|}{$B W=10 \mathrm{kHz}$} & \multicolumn{3}{|c|}{$\mathrm{BW}=100 \mathrm{kHz}$} \\
\hline & Star & Tree & Mesh & Star & Tree & Mesh \\
\hline $\begin{array}{c}\text { Throughput } \\
\text { (bits/sec) }\end{array}$ & 31300 & 45850 & 29200 & 31300 & 45850 & 29200 \\
\hline $\begin{array}{c}\text { Load } \\
\text { (bits/sec) }\end{array}$ & 32400 & 45850 & 29100 & 32400 & 45850 & 29100 \\
\hline $\begin{array}{c}\text { Data Traffic } \\
\text { Sent (bits/sec) }\end{array}$ & 36500 & 51640 & 32790 & 36500 & 51640 & 32790 \\
\hline $\begin{array}{c}\text { Data Traffic } \\
\text { Received } \\
\text { (bits/sec) }\end{array}$ & 510000 & 722900 & 458000 & 510000 & 722900 & 458000 \\
\hline \multicolumn{7}{|c|}{ Network Size $=\mathbf{5 0}$ nodes } \\
\hline & \multicolumn{3}{|c|}{$B W=10 \mathrm{kHz}$} & \multicolumn{3}{|c|}{$B W=100 \mathrm{kHz}$} \\
\hline & Star & Tree & Mesh & Star & Tree & Mesh \\
\hline $\begin{array}{c}\text { Throughput } \\
\text { (bits/sec) }\end{array}$ & 108500 & 181500 & 113600 & 108500 & 186500 & 122200 \\
\hline $\begin{array}{c}\text { Load } \\
\text { (bits/sec) }\end{array}$ & 109600 & 193800 & 109100 & 109600 & 201300 & 115200 \\
\hline $\begin{array}{c}\text { Data Traffic } \\
\text { Sent (bits/sec) }\end{array}$ & 123400 & 218200 & 123000 & 123400 & 221600 & 129900 \\
\hline $\begin{array}{c}\text { Data Traffic } \\
\text { Received } \\
\text { (bits/sec) }\end{array}$ & 6050000 & 9595000 & 5600000 & 6050000 & 123400 & 1299000 \\
\hline
\end{tabular}

\section{CONCLUSION}

In order to optimize the performance of PHY layer parameters for the Zigbee network work has been done in this paper. In order to establish reliable short range communication frequency band, data rate and $\mathrm{BW}$ have been optimized. In doing so simulation scenario has been created for mesh, tree and star topologies using OPNET modeler.

Performance evaluation has been done in terms of throughput, load, data traffic sent/received and it was observed that tree topology outperforms both in terms of throughput and delay at $2.4 \mathrm{GHz}$. However it was observed that all the three topologies performs better at $2.4 \mathrm{GHz}$ in comparison to $915 \mathrm{MHz}$. Further the effect of data rate has been evaluated by varying at $50 \mathrm{kbps}, 100$ kbps and $150 \mathrm{kbps}$. It was observed that $100 \mathrm{kbps}$ data rate give optimal performance for 15 nodes network but performance of star topology show minor variation in performance. Considering BW as an important resource in the Zigbee network performance evaluation has also been done by varying the BW. It was observed that the network throughput increases with increase in BW. This performance gain can only be achieved for large size networks. Thus the results obtained here, can be used as ready reference for the design engineering implementing Zigbee network based prototypes.

\section{REFERENCES}

[1] Muthu Ramya.C, Shanmugaraj.M, Prabakaran.R. Study on Zigbee Technology, IEEE, 3rd International Conference on Electronics Computer Technology (ICECT), Vol. 6, Kanyakumari, India, 8-10 April 2011.

[2] Jin-Shyan Lee, Yu-Wei Su, and Chung-Chou Shen. A
Comparative Study of Wireless Protocols:Bluetooth, UWB, ZigBee, and Wi-Fi. The 33rd annual conference of the IEEE industrial electronics society (IECON), Taipei, Taiwan, 5-8 November, 2007.

[3] Manpreet, Jyoteesh Malhotra, Zigbee:Cureent Status and Future Scope, International conference on computer and cmputational sciences (ICCCS), IEEE, Greater Noida, India, , 27-29 January 2015.

[4] Manpreet, Jyoteesh Malhotra. Simulation analysis of tree and mesh topologies in Zigbee network, Internation journal of grid and distributed computing, Vol.1, No. 1, pp. 81-92, 2015.

[5] Sukhvinder Singh Bamber and Ajay Kumar Sharma. Simulative Performance Evaluation of 802.15.4 with Different Modulations for Wireless Sensor Networks, International journal of computer network and communications, Vol. 2, No. 6, November 2010.

[6] Khaled Shuaib, Maryam Alnuaimi, Mohamed Boulmalf, Imad Jawhar, Farag Sallabi and Abderrahmane Lakas. Performance. Evaluation of IEEE 802.15.4: Experimental and Simulation Results, Journal of Communications, Vol. 2, No. 4, June 2007.

[7] Er. Anamika Vatsal, Prof. Mehajabeen Fatima. Impact of Frequency Offset on Interference between Zigbee and Wifi for Smart Grid Applications. IOSR Journal of Electronics and Communication Engineering (IOSRJECE).Volume 7, Issue 5, PP 59-64, September-October, 2013.

[8] Wireless Networking Technology Rackley 2007

[9] Wireless Comm and Networking vijay 2007

[10] The network simulator- OPNET, http:// www.opnet.com/ solutions/network_rd/modeler.html.

[11] The network simulator-ns-2, http://www.isi.edu /nsnam /ns/.

[12] Ke Dong, Dr. Venkatesha Prasad Ranga Rao,Huaizhou Shi "Performance and Fairness Enhancement in ZigBee Networks", Masters Thesis, Delft University of Technology, Oct 2011. 


\section{Authors' Profiles}

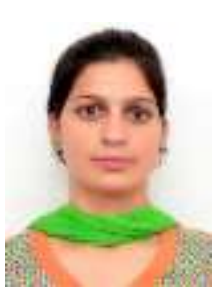

Manpreet lives in Jalandhar, Punjab, INDIA. She has been completed B.Tech in Computer Science \& Engineering in 2008 from Punjab Technical University, Jalandhar She is pursuing M.Tech in Computer Science \& Engineering from Guru Nanak Dev Univesity Regional Campus Jalandhar, Punjab, INDIA. She has been submitted thesis work on Zigbee Network Topologies supervised by Dr. Jyoteesh Malhotra (Head of Department of Electronics and Computer Science \& Engineering). She qualified GATE 2014 and GATE 2015. She has paper published in IEEE conferences and other journals. She has work experience in software development and her thesis work focused the wireless personal area network (Zigbee).

Dr. Jyoteesh Malhotra is Head of Department in Computer Science \& Engineering and Electronics Department. He has been done $\mathrm{Ph}$. D in electrical and communication; He is Gold medalist in masters in technology. He has more than 20 years work experience. His specialization is in Wireless / Optical Comm. Systems and Networks. He has more than 100 publications of research papers and books.

How to cite this paper: Manpreet, Jyoteesh Malhotra,"On the Selection of Physical Layer Optimized Network Topologies for the Zigbee Network", International Journal of Information Technology and Computer Science(IJITCS), Vol.8, No.1, pp.84-94, 2016. DOI: 10.5815/ijitcs.2016.01.10 